\title{
DESASTRES NATURAIS NO ESTADO DE SANTA CATARINA - 1998 A 2019
}

NATURAL DISASTERS IN THE STATE OF SANTA CATARINA - 1998 TO 2019

RACHEL FAVERZANI MAGNAGO, Dr. |UNISUL

PATRÍCIA MEDEIROS | UNISUL

RODRIGO PAULO RAIMUNDO |IFSC

SUSANA CLAUDETE COSTA, M.SC. | DEFESA CIVIL

\begin{abstract}
RESUMO
O crescimento populacional e o aumento da densidade demográfica bem como o efeito das mudanças climáticas e os eventos climatológicos vem aumentando a vulnerabilidade das comunidades e elevando a discussão sobre resiliência nas cidades, principalmente as comunidades que vivem em áreas de risco. Diante disso, foram analisados os dados de desastres naturais dos grupos climatológico, geológico, hidrológico, meteorológico e biológico que atingiram o estado de Santa Catarina de 1998 a 2019. A análise incluiu informações de níveis de incidência de ENSO. Inicialmente foi identificado que os desastres naturais no estado de Santa Catarina ocorrem devido à hidrologia e meteorologia típica da região, sendo que foram decorrentes principalmente de acúmulos de chuva e adensamentos populacionais mais volumosos. O histórico de desastres naturais contribui no monitoramento para visualização das consequências no Estado, e contribuí com informação e conscientização da população, para prevenir e preparar a população para enfrentamento de desastres naturais.
\end{abstract}

PALAVRAS CHAVE: Desastre Natural; ENSO; Santa Catarina

\begin{abstract}
Population growth and increased population density, as well as the effect of climate change and climatological events, have increased the vulnerability of communities and raised the discussion about resilience in cities, especially communities living in areas at risk. Therefore, the data on natural disasters of the climatological, geological, hydrological, meteorological and biological groups that affected the state of Santa Catarina from 1998 to 2019 were analyzed. The analysis included information on ENSO incidence levels. Initially, it was identified that natural disasters in the state of Santa Catarina occur due to hydrology and meteorology typical of the region, and they were mainly due to accumulations of rain and more massive population densities. The history of natural disasters contributes to the monitoring to visualize the consequences in the State, and contributes with information and awareness of the population, to prevent and prepare the population to face natural disasters.
\end{abstract}

KEY WORDS: Natural disasters; ENSO; Santa Catarina 


\section{INTRODUÇÃO}

A agenda internacional entre países é marcada por prioridades, como o futuro do trabalho, o combate à pobreza, a promoção da paz entre nações e as mudanças climáticas. Registrado historicamente na conferência de Estocolmo em 1972 os efeitos das mudanças climáticas como anormalidade no aumento de temperatura do planeta afetando a vida das pessoas (Marcatto e Lima, 2013).

Além disto, tem-se o aumento da densidade demográfica em áreas urbanas influenciando ambientes naturais e ampliando a vulnerabilidade, elevando a prioridade de discussão sobre Risco de Desastres (Hamdan, 2015; Moraes e Borja 2005; Silva e Rodriguez 2011).

Em 1999, foi criado o Escritório das Nações Unidas para Redução de Riscos de Desastres com a missão de habilitar sociedades para resistirem aos perigos e aos desastres tecnológicos e ambientais, com o objetivo de reduzir perdas humanas, econômicas e sociais (UNDRR, 2021).

A agenda 2030, escrita com a colaboração de 169 países, elenca os objetivos de desenvolvimento sustentável (ODS) para o planeta até 2030. Esses objetivos pretendem promover uma desaceleração das mudanças climáticas, por um futuro mais adequado a vida humana. São dezessete ODS, dos quais três abordam entre os seus indicadores, especificamente o tema desastres, sendo ODS 1 relacionado a erradicação da pobreza, ODS 11 sobre cidades e comunidades sustentáveis e ODS 13 denominada ação contra a mudança global do clima, meteorológico e a desastres naturais (UNDRR, 2021).

No Brasil, juntamente ao Instituto de Pesquisas Econômica Aplicada e baseado na Agenda 2030 Global, o Ministério do Planejamento, Desenvolvimento e Gestão, desenvolveu em 2018 os Objetivos de Desenvolvimento Sustentáveis Nacionais, descrevendo as metas brasileiras. Os ODS nacionais relacionados diretamente ao tema Redução de Risco a Desastres são: 1. Erradicação da Pobreza (Meta 1.5 - construir a resiliência dos pobres e daqueles em situação de vulnerabilidade, e reduzir a exposição e vulnerabilidade destes a eventos extremos relacionados com o clima e outros choques, e desastres econômicos, sociais e ambientais); 11 . Cidades e comunidades sustentáveis (Meta 11.5: Até 2030, reduzir significativamente o número de mortes e o número de pessoas afetadas por desastres naturais de origem hidrometeorológica e climatológica, bem como diminuir substancialmente o número de pessoas residentes em áreas de risco e as perdas econômicas diretas causadas por esses desastres em relação ao produto interno bruto, com especial atenção na proteção de pessoas de baixa renda e em situação de vulnerabilidade. Meta 11.b: Até 2030, aumentar significativamente o número de cidades que possuem políticas e planos desenvolvidos e implementados para mitigação, adaptação e resiliência a mudanças climáticas e gestão integrada de riscos de desastres conforme o Marco de SENDAI); 13. Combate as mudanças climáticas (Meta 13.1: Ampliar a resiliência e a capacidade adaptativa a riscos e impactos resultantes da mudança do clima e a desastres naturais).

Entre as metas brasileiras, se destaca a prioridade de ação voltada para pessoas em situação de vulnerabilidade, residindo em áreas de risco. Países em desenvolvimento são mais vulneráveis a desastres, há relação entre risco a desastres com a pobreza, no entanto, as consequências podem ser reduzidas quando a população está preparada (Fay, Ghesquiere e Solo, 2003). Almeja-se diminuir o impacto dos desastres naturais, que afetam as pessoas e geram perdas humanas e/ou econômicas, através da construção da resiliência das cidades e comunidades.

Ainda a meta 11.b se refere a adoção de medidas previstas no Marco de Sendai para Redução de Riscos a Desastres (SFDRR) de 2015 a 2030, acordo firmado entre os 187 países em Sendai, no Japão em 2015. Em conjunto, os países definiram ser necessário realizar ações de prevenção e preparação, não apenas de resposta (Mendonca; Silva Rosa; Bello, 2019).

Para uma devida prevenção e preparação é fundamental ter-se conhecimento dos desastres que ocorrem em uma determinada região, bem como os fatores que colaboram para estes acontecerem, ou ainda, que os intensifiquem. Então deve-se desenvolver conscientização e educação ambiental dos habitantes, e políticas públicas com base nos desastres ambientais e/ou tecnológicos ocorridos em uma determinada região com escala temporal (Souza, 2016).

A codificação de desastres diz respeito à tipificação dos desastres, já que o desastre expressa o resultado de um evento adverso, em consequência. Para fins de tipificação, a Codificação Brasileira de Desastres define-se em função dos eventos adversos, causadores destes, segue classificação criteriosa, iniciando pela categoria, natural e tecnológico. Neste trabalho, foi considerado a categoria natural, sendo os grupos geológicos, hidrológico, climático e biológico (Defesa Civil, 2020).

O Estado de Santa Catarina, destaca-se a alta prevalência de desastres hidrológicos como as inundações e geológicos como o movimento de massa. Uma dessas catástrofes ocorreu no Vale do Itajaí em 2008, resultando em perdas econômicas e sociais significativas devido 
a chuvas intensas, enchentes e múltiplos deslizamentos, que resultaram em 135 mortos e milhares de afetados em 60 cidades (Tominaga, Santoro e Amaral, 2009).

Apesar do SFDRR indicar a prevenção de desastres a partir de ações educativas, assim como em outros lugares do mundo, em Santa Catarina, as ações focadas na redução de risco foram motivadas após desastres fatídicos, como o sofrido pela cidade de Blumenau em 2008. Apenas em 2013, foram desenvolvidas ações educativas formais e contínuas sobre redução de risco a desastres na região (Oliveira, Ouriques e Correia, 2018; Silva et al., 2018).

Mesmo sendo lamentável estes acontecimentos apontam para eventos cíclicos e com aumento significativo trazendo graves consequências para a sobrevivência, dos seres humanos, principalmente aos mais pobres e vulneráveis (Banco Mundial, 2012). Mas, para melhor compreensão, preparação e enfrentamento faz-se necessário uma análise temporal e ainda se as mudanças climáticas (meta13.1) corroboram com desastres naturais no estado de Santa Catarina.

Neste contexto, o objetivo foi analisar os desastres naturais ocorridos no período de 1998 a 2019 do estado de Santa Catarina registrados na Defesa Civil. Analisar o histórico de desastres naturais pode ser uma ferramenta de monitoramento para visualização das consequências da situação hidrológica, meteorológica e climática no Estado, também pode ser um meio de informação e conscientização da população com dados históricos de eventos, para prevenir e preparar a população para enfrentamento de desastres naturais.

\section{MATERIAIS E MÉTODOS}

O estudo foi realizado no estado de Santa Catarina, localizado ao sul do Brasil nas latitudes $25^{\circ} 57^{\prime} 41^{\prime \prime S}$ e $29^{\circ} 23^{\prime} 55^{\prime \prime S}$ e 48 $19^{\prime} 37^{\prime \prime}$ W e $53^{\circ} 50^{\prime} 00^{\prime \prime} W$, possui área total de 95.736,165 $\mathrm{km} 2$, se estende das fácies praiais às Escarpas da Serra do Mar e dos Campos Gerais que dividem o estado entre uma região de planalto a oeste e a planície litorânea a leste (Bortoluzzi 1987, Short \& Klein, 2016).

Conforme a classificação de Köppen, Santa Catarina possui um clima mesotérmico úmido sem estações secas, abrangendo também o clima oceânico. Devido à sua localização e relevo, aliados à atuação da Massa de ar Polar Atlântica e da Massa Tropical Atlântica, Santa Catarina apresenta uma distribuição pluviométrica regular durante o ano, com uma média de cerca de 1500 mm/ano (Andrade, Baro e Nery 1999), sendo os maiores índices de precipitação observados próximo à encosta da Serra Geral.
Segundo a Agência Nacional de Águas os rios que drenam o território estadual de Santa Catarina integram três grandes Regiões Hidrográficas - a Região Hidrográfica do Paraná, a Região Hidrográfica do Uruguai e a Região Hidrográfica Atlântico Sul. A rede hidrográfica catarinense tem na Serra Geral o principal divisor de águas que forma os dois sistemas independentes de drenagem do território estadual: o sistema integrado da Vertente do Interior, compreendendo 07 bacias que integram a bacia ParanáUruguai, e o sistema da Vertente Atlântica, formado por um conjunto de 11 bacias isoladas que fluem para leste, desaguando diretamente no Atlântico. Assim, a rede hidrográfica catarinense possui um total de 18 bacias hidrográficas consideradas de rios principais (SDS, 2021).

Na Figura 1, o mapa das cidades nas regiões hidrográficas do estado de Santa Catarina.

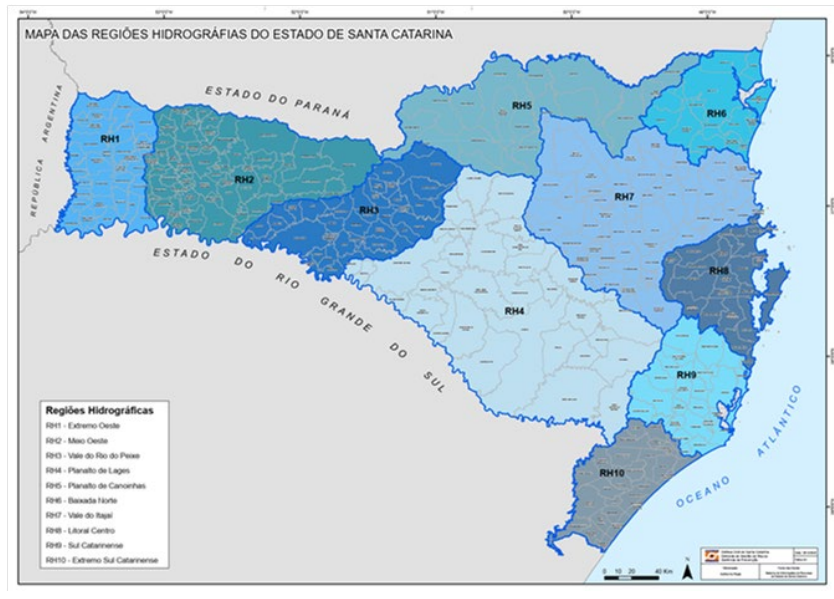

Figura 01 - Mapa das cidades nas regiões hidrográficas do estado de Santa Catarina. Fonte: Adaptado do Sistema de Informações de Recursos do Estado de Santa Catarina.

Os dados de desastres foram coletados da plataforma online da Defesa Civil de Santa Catarina no período de 1998 a 2019, esses foram organizados em uma planilha Excel.

Os dados históricos de 1998 a 2019 de eventos de El Niño e La Niña mensais foram obtidos da plataforma online da Administração Oceânica e Atmosférica Nacional (NOAA).

\section{RESULTADOS E DISCUSSÕES}

Nesse trabalho, foram considerados os desastres na categoria natural, nos grupos climatológico, geológico, hidrológico, meteorológico e biológico.

Na Figura 2 têm-se 5051 ocorrências de desastres naturais incluindo climatológico, geológico, hidrológico, meteorológico e a somatória anual do período de 1998 a 2019 a partir dos dados registrados na Defesa Civil de Santa Catarina. Os desastres naturais hidrológicos (2271) 
prevalecem sobre climáticos (1372) e meteorológicos (1363), sendo que os geológicos (45) foram números bem menores que os outros desastres entre 1998 a 2019.

Ainda na Figura 2, o número de desastres naturais do estado de Santa Catarina no período de 1998 a 2008 foi menor que do período de 2009 a 2019, sendo 2197 e 2794, respectivamente. No período de 1998 a 2007 prevaleceram registros de desastres de origem climatológico (seca) e no período de 2008 a 2019 os registros foram de origem hidrológica (inundação, enxurrada e alagamento). $\mathrm{O}$ ano 2011 foi de maiores registros de ocorrências de desastres naturais no estado de Santa Catarina no período estudado, num total de 685, sendo 524 hidrológico, 109 meteorológico, 46 climatológico e 6 geológico, e os anos 2009 e 2010 também apresentaram importantes registros de desastres hidrológicos.

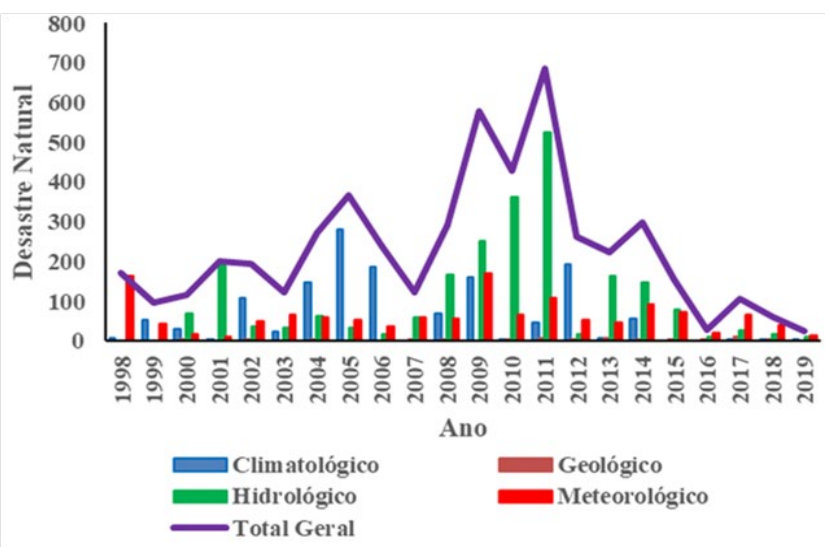

Figura 02 - Desastres naturais climatológico, geológico, hidrológico, meteorológico e a somatória anual do período de 1998 a 2019.

Fonte: Adaptado da Defesa Civil do Estado de Santa Catarina

Na Figura 3, tem-se o acumulado de desastres naturais climatológicos, geológicos, hidrológicos e meteorológicos mensais no período de 1998 a 2019.

Na Figura 3 têm-se maiores ocorrências de desastres naturais no período de chuvas do estado de Santa Catarina, comumente nas estações de primavera (meses de setembro, outubro e novembro) e verão (meses de dezembro, janeiro e fevereiro). Nestes meses observa-se a ocorrência de desastres naturais, estando de modo geral, associados a precipitação intensa ou a ausência desta. Os meses com maiores registros de desastres naturais foram janeiro, setembro e outubro, com total de 777, 588 e 472, respectivamente, mas também há ocorrências nas outras estações do ano. $O$ número de registro não necessariamente traduz as consequências do desastre, mas alerta para o período de maior frequência, e então para a preparação da população. Por exemplo, em outubro de 2008 na região o Vale do Itajaí ocorreu maior número de óbitos já registrados no estado de Santa Catarina. Além disso, o registro do desastre nem sempre é a causa de óbitos, em 2008, a causa de óbitos foi o desastre geológico, visto que o movimento de massa foi a principal causa das mortes.

A Figura 3 expressa também os desastres naturais geológicos por mês de 1998 a 2019, sendo que de 45 desastres registrados neste período foram 18 movimentos de massas, 01 erosão laminar, 06 erosões, 08 erosões fluviais e 12 erosões costeiras. As erosões fluviais causadas pelas águas dos rios provocam desgaste e/ou removem porções do solo das margens dos rios, provocando um deslizamento de terra, são geralmente intensificadas por erosões pluviais, dependendo da intensidade e/ou frequência podendo mudar o curso de um rio. $\mathrm{Na}$ erosão costeira a praia perde mais sedimentos do que recebe, deste modo o recuo da linha de costa é uma consequência deste processo (SDS, 2021). Em situações extremas, como de segurança à população e ainda perdas econômicas que comprometam a renda da população medidas de contenção e reconstrução devem ser tomadas, tais como enrocamentos, gabiões e colchões Reno.

Também na Figura 3 tem-se que a seca é o principal evento oriundo dos 1372 desastres naturais climatológicos entre 1998 e 2019, sendo que foram 8 exaurimentos de recursos hídricos e os demais resultantes de estiagem. Registros de condições de seca são especialmente importantes para as regiões de Santa Catarina, pois apresentam atividades econômicas dependentes dos recursos hídricos e afetam particularmente os municípios que dependem fortemente da agricultura, incluindo subsistência e pequenas/médias propriedades que fornecem seus produtos para serem beneficiados pelas empresas agroindustriais (SDS, 2021). Apesar de a estiagem ser um evento cíclico no estado, ainda as iniciativas, tais como sistemas de captação, reservatório e uso de água da chuva, precisam ser melhor implementadas, pois a estiagem que assola Santa Catarina desde 2019, mostra-se ainda mais severa que em anos anteriores, a seca impacta a agropecuária principalmente de produtores do Oeste, MeioOeste, Extremo-Oeste e Planalto Sul, e também comprometendo abastecimento de água. $O$ ano de 2020 finalizou com 9 rios em situação de estiagem em Santa Catarina, a situação foi considerada de emergência Rio da Antas (Mondaí), Rio Itajaí-Açu (Alfredo Wagner, Blumenau, Chapadão do Lageado e José Boiteux), Rio Tijucas (São João Batista), Rio do Chapecó (Coronel Freitas e São Carlos), Rio do Peixe (Joaçaba e Tangará), Rio Jacutinga (Concórdia) e Rio Camboriú (Camboriú) e em outros 6 a situação de seca está sob alerta. 
Ainda na Figura 3, do total de 1363 desastres naturais meteorológicos entre 1998 a 2019, 1276 foram tempestades, 38 tempestades extremas e 49 sistemas de Grande Escala/Escala regional. O investimento para o monitoramento e execução de obras de infraestrutura para conter impactos negativos é uma das tentativas do Estado para minimizar as perdas humanas e econômicas (FIESC, 2018). Dessa forma, o monitoramento e alerta traz ações de comunicação com melhor preparação das comunidades.

Finalizando, na Figura 3, do total de 2271 desastres naturais hidrológicos no período do estudo, foram 2101 enxurradas, 106 inundações e 64 alagamentos. Trazendo grande perda econômica e humana muitas vezes pela ocupação desordenada em áreas de risco.

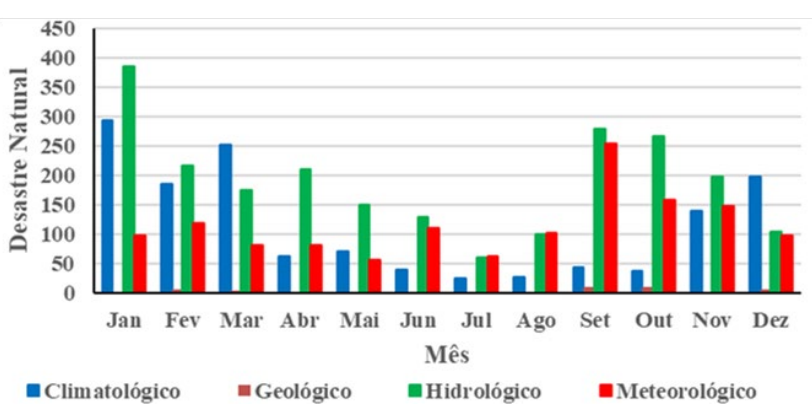

Figura 03 - Soma de desastres naturais climatológico, geológico, hidrológico, meteorológico mensais no período de 1998 a 2019.

Fonte: Adaptado da Defesa Civil do Estado de Santa Catarina.

Os desastres naturais registrados no estado de Santa Catarina estão associados a um complexo e variado fatores hidrometeorológicos e climatológicos (regime de chuvas, temperatura, pressão, massas de ar, latitude, altitude, vegetação, relevo), sendo sensível a alterações de cada um deles e outros fenômenos, sendo que os fatores estão interligados, influenciando-se mutualmente. No entanto, alguns acontecimentos climáticos são classificados como anomalias, representando alterações no sistema atmosférico e provocando mudanças em várias partes do planeta. Os fenômenos climáticos de El Niño (anomalias positivas da temperatura da superfície do mar no Oceano Pacífico) e La Niña (anomalias negativas da temperatura da superfície do mar no Oceano Pacífico) são observados por variáveis meteorológicas, o que permite acompanhar as mudanças climáticas e seus possíveis efeitos em determinadas regiões (Moura et al., 2019).

ENSO é o modo dominante de variabilidade acoplada atmosfera-oceano em escalas de tempo interanuais em várias regiões do mundo (Trenberth e Stepaniak, 2001). Uma das regiões com maiores impactos associados a precipitações extremas e eventos ENSO, é o Sul da América do Sul (Penalba; Rivera, 2016).
Na figura 4 tem-se a média anual do fenômeno El Niño-Oscilação Sul (ENSO) e a somatória de desastres naturais anuais dos grupos geológico, climatológico, meteorológico e hidrológico.

De modo geral, a Figura 4 demonstra que nos anos de neutralidade do ENSO ocorreram os menores registros de desastres naturais no estado de Santa Catarina. Os anos de 2009 e 2011 que apresentaram recordes de desastres naturais em Santa Catarina, 578 e 685, foram respectivamente precedidos de anos de fenômeno climático La Niña, com déficits de precipitação, e com El Niño no ano de 2009 (Grimm et al., 2000; Grimm et al., 2009).

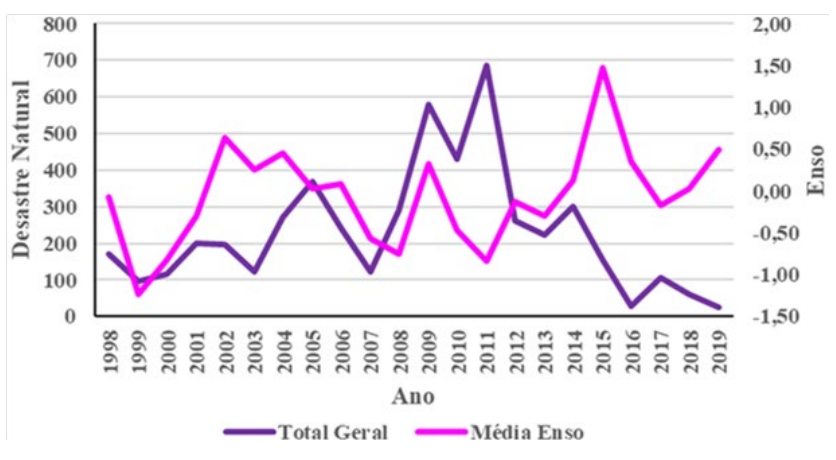

Figura 04 - Soma anual de desastre natural e média anual de ENSO de 1998 a 2019. Fonte: Adaptado do Sistema de Informações de Recursos do Estado de Santa Catarina.

Na Figura 5 tem-se a soma anual de desastre natural geológico (A), climático (B), meteorológico (C) e hidrológico (D) e média anual de ENSO de 1998 a 2019.

O desastre geológico é definido por um processo geológico natural, mas que causa algum dano à vida, dano social e/ou econômico. O desastre climatológico é caracterizado por conjunto de eventos ao longo de um tempo. Já o Meteorológico, os eventos são bruscos e extremos em um período muito curto (Parizzi, 2014). O desastre, seja qual for o fenômeno natural responsável em conjunto com o intenso processo de urbanização pode trazer consequências duradouras na região afetada (Tominaga, Santoro e Amaral, 2009).

Os estudos pioneiros de Ropelewski e Halpert (1987) e Ropelewski e Halpert (1986), identificou um efeito ENSO claro nos padrões de precipitação sobre o sul da América do Sul, caracterizado por um aumento na precipitação sobre o centro-leste da Argentina, Uruguai e Sul do Brasil durante o verão após o desenvolvimento das condições do El Niño.

Com foco na América do Sul e oceanos circunvizinhos, Aceituno (1988) também mostrou que as mudanças relacionadas ao ENSO na circulação em grande escala levam a uma vasta diversidade de regimes anômalos de precipitação regional. Esse resultado foi verificado posteriormente por Grimm et 
al. (2000, 2009), que realizaram uma análise da variabilidade da precipitação associada às fases El Niño e La Niña através de uma abordagem regional. O sinal mais importante geralmente ocorre durante o verão do hemisfério sul e o outono após o ano de ocorrência dos eventos El Niño e durante a primavera após os anos La Niña. Estes padrões de precipitação parecem explicar os observados na Figura 3 e Figura 4.

Penalba et al. (2005) analisaram a probabilidade de ultrapassar a mediana durante às duas fases do ENSO, mostrando coerência entre a ocorrência do El Niño (ou La Niña) e o excesso de precipitação (ou déficit) sobre o centro-leste da Argentina e sul do Brasil. No entanto, este estudo mostrou um alto grau de variabilidade regional na precipitação relacionada ao ENSO, que deve ser considerado nas previsões de precipitação em grandes áreas.

Na Figura 5 pode ser observado efeito do ENSO, de forma direta ou indireta, nos desastres ocorridos em Santa Catarina. Pode ser observado na Figura 5 nos anos de La Niña ocorreram registros de desastres geológicos (Figura $5 \mathrm{~A}$ ) e climático (Figura 5B), sendo a seca que atinge mais pessoas e atividades no estado.

Desastres climático-meteorológicos podem ser intensificados tanto com El Nino como com La Niña, dando destaque no estado para as tempestades (tornados, tempestade de raios, granizo, chuvas intensas e vendaval) e temperaturas extremas (onda de frio e geadas) (Souza et al., 2005).
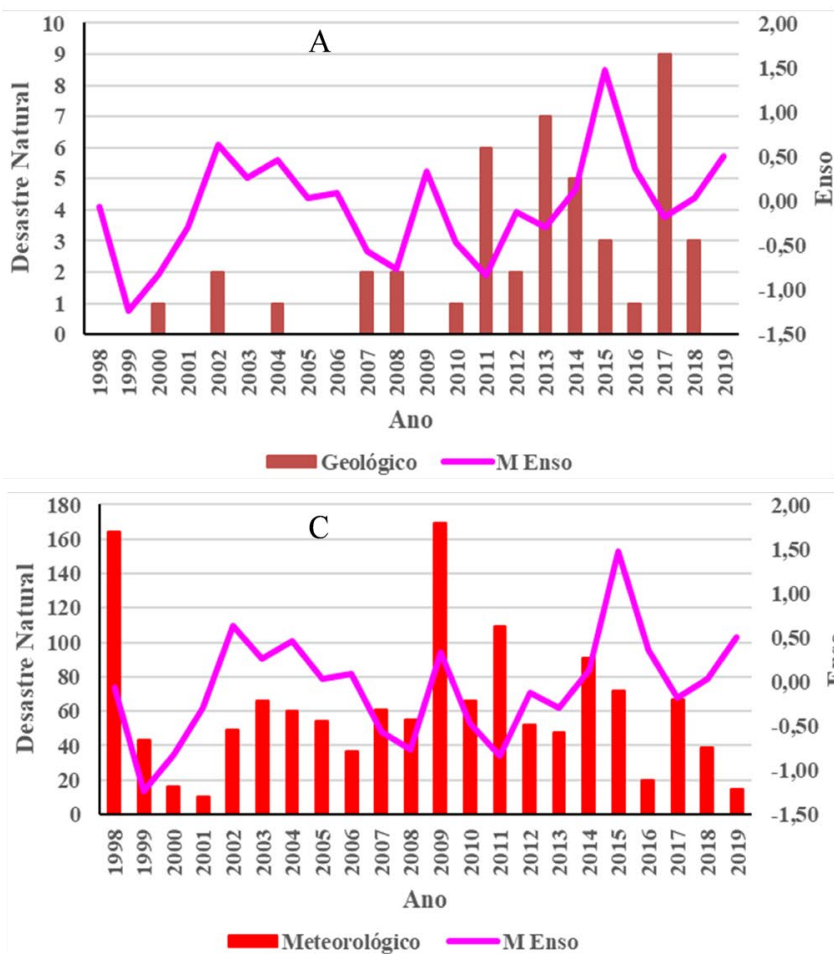

Os impactos dos eventos El Niño e La Niña foi evidente no setor hidrológico (Boulanger et al., 2005) sobre a sul da América do Sul e particularmente sobre o extremo sul do Brasil. O excesso de precipitação associado aos eventos El Niño nos meses de verão, no estado de Santa Catarina, aumentando o teor de água no solo e contribuindo para o acúmulo, parece contribuir a desastres ambientais em meses subsequentes. Os transbordamentos e inundações de rios no estado de Santa Catarina parecem estar relacionados pelo volume de chuvas ao longo do período de primavera e verão (Figura 3).

Os desastres na sua maioria são acontecimentos de complexa análise, que devem ser considerados os dados hidrológicos em conjunto com as informações geológicas e com os dados meteorológicos, ainda não pode ser ignorado fatores antrópicos, a combinação desses podem resultar em desastres hidrológicos (Figura 5D) como as inundações e geológi$\cos$ (Figura 5A) como movimento de massa.

Em relação às tendências de precipitação, Barros et al. (2008) demonstraram que metade das tendências anuais no sul do Brasil estavam associadas à fase El Niño. Mesmo quando há evidência da existência de uma região central em relação ao sinal de ENSO nos padrões de precipitação, outras áreas do sul da América do Sul mostraram uma modulação nas variáveis hidroclimáticas associadas aos eventos El Niño e La Niña.

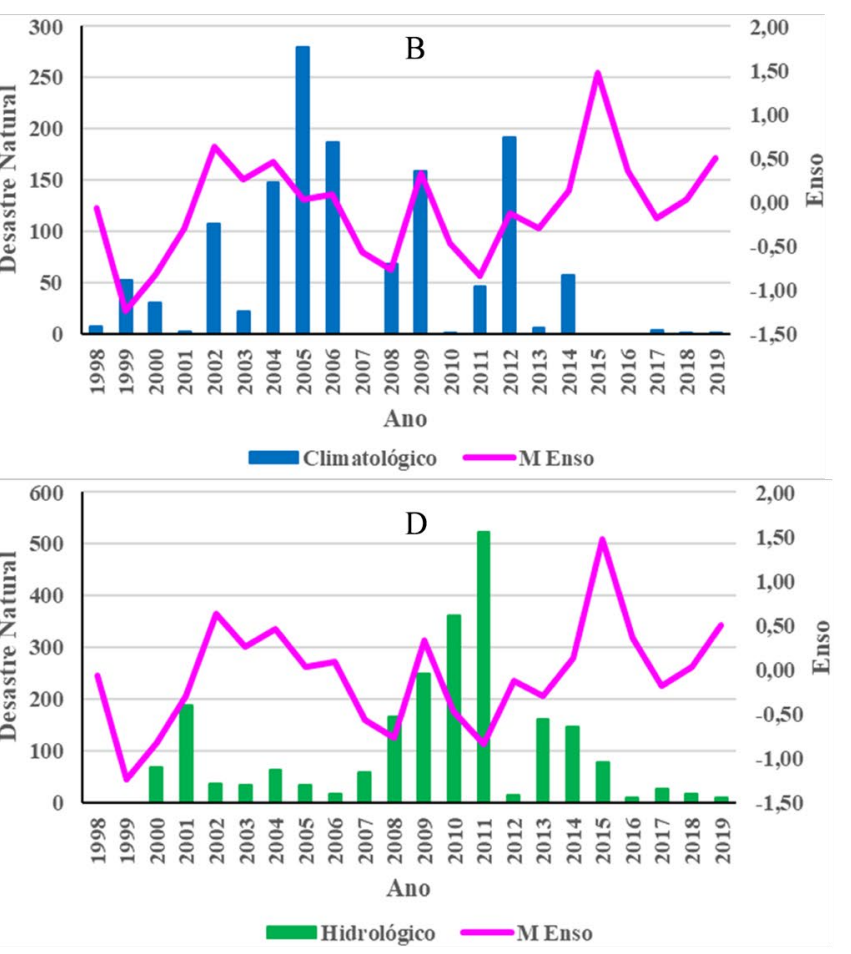

Figura 05 - Soma anual de desastre natural geológico (A), climático (B), meteorológico (C) e hidrológico (D) e média anual de ENSO de 1998 a 2019. Fonte: Adaptado do Sistema de Informações de Recursos do Estado de Santa Catarina 
Na Figura 6 a partir das cidades das ocorrências de desastres naturais identificaram-se as regiões hidrográficas do estado de Santa Catarina, sendo que uma cidade pode situar-se em mais de uma região hidrográfica.

Na Figura 6, pode-se observar que desastres naturais hidrográficos ocorreram em todas as regiões do estado de Santa Catarina no período de 1998 a 2019, sendo de maior frequência no Vale do Itajaí. As quatro regiões hidrográficas $(\mathrm{RH})$ que se destacam pela ocorrência de desastres naturais foram o Vale do Itajaí ( $\mathrm{RH} 7)$, Meio Oeste $(\mathrm{RH} 2)$, Planalto de Lages (RH 4) e Vale do Rio do Peixe (RH3), em $\mathrm{RH} 7$ e $\mathrm{RH} 4$ predominou desastres hidrológicos, enquanto $\mathrm{RH} 2$ e RH 3 foram os desastres naturais climatológicos.

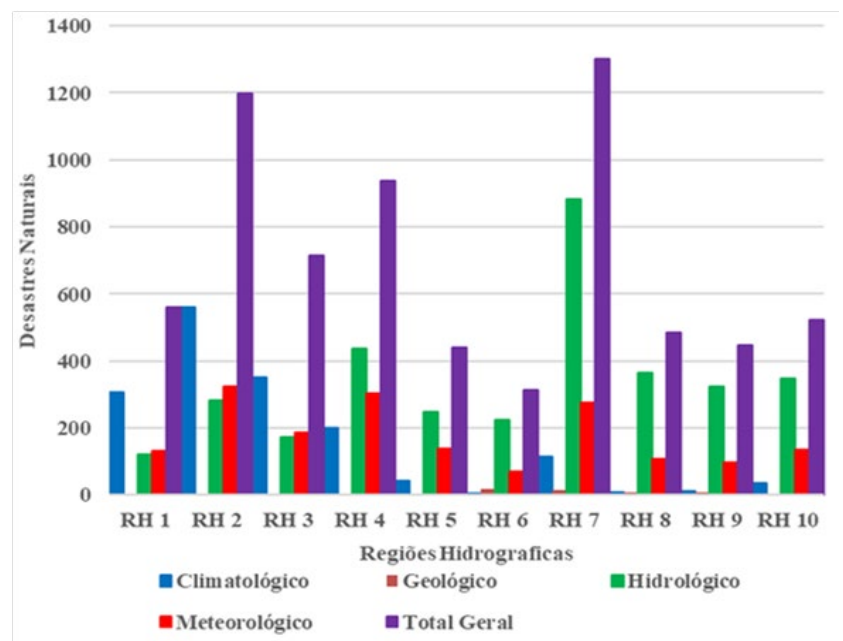

Figura 06 - Regiões hidrográficas do estado de Santa Catarina das ocorrências de desastres naturais de 1998 a 2019.

Fonte: Adaptado do Sistema de Informações de Recursos do Estado de Santa Catarina.

A principal bacia formadora da $\mathrm{RH} 7$ é a bacia do rio Itajaí-Açu, cujo curso pode ser subdividido em três principais segmentos: (i) Alto Itajaí-Açu: trecho de $26 \mathrm{~km}$ de extensão, com início na confluência das sub-bacias do Itajaí do Sul e Itajaí do Oeste, no município de Rio do Sul, até salto de Pilões, a montante da foz do Itajaí do Norte; (ii) Médio Itajaí-Açu: trecho de $83 \mathrm{~km}$ de extensão com início no salto dos Pilões e segue até o salto de Weissbach, nas proximidades de Blumenau; e (iii) Baixo Itajaí-Açu: trecho de 80 km de extensão que inicia no salto de Weissbach chegando até a desembocadura no Oceano Atlântico; podem ainda ser definidas no contexto desta bacia sete sub-bacias principais: Benedito, Itajaí do Norte (ou Hercílio); Itajaí do Oeste; Itajaí do Sul; Itajaí-Mirim, Itajaí-Açu e Luís Alves. No total, a bacia do Itajaí concentra um contingente superior a 1.240.000 pessoas. A população urbana, em torno de 1.040 .000 habitantes está distribuída em 49 sedes municipais, sendo Blumenau o principal polo econômico regional. A ocorrência de desastres naturais hidrológicos (inundações, enxurradas e alagamentos) periódicas tem sido considerado um dos maiores problemas no vale do rio Itajaí-Açu. Cabe destacar também a região inclui drenagens independentes que fluem em direção ao oceano, com destaque para o rio Camboriú, cuja qualidade das águas influencia diretamente as condições de balneabilidade do Balneário do Camboriú, principal destino turístico catarinense.

A RH 4 é a maior Região Hidrográfica em extensão de Santa Catarina (22.766 km2), integrando duas bacias hoje consideradas de domínio da União: do rio Canoas, que corresponde a área de $14.908 \mathrm{~km} 2$ e do rio Pelotas com área de 7.277 km2 em território catarinense. O rio Canoas tem como afluentes, entre outros, o rio Correntes e o Caveiras nas margens direita e esquerda, respectivamente; $\mathrm{O}$ rio Pelotas serve de limite entre os Estados de Santa Catarina e Rio Grande do Sul. No seu trajeto, recebe a contribuição dos rios Pelotinhas e São Mateus pela margem direita; A partir da união dos rios Canoas e Pelotas forma-se o rio Uruguai. No total, encontram-se na bacia do rio Canoas as sedes de 28 municípios, comportando uma população urbana superior a 340 mil habitantes, com destaque para Lages, Fraiburgo, Curitibanos e Campos Novos.

As duas principais bacias hidrográficas que compõe a $\mathrm{RH} 2$ são a do rio Chapecó $(8.300 \mathrm{~km} 2)$ e a do rio Irani (1.595 km2) e outros, com área total desta região hidrográfica é de 11.289 km2. A bacia do rio Chapecó, com 236 mil pessoas, drena 42 sedes urbanas, onde vivem cerca de 138.000 habitantes. Essas cidades, em geral de pequeno e médio porte, como Quilombo, Jardinópolis, estão situadas principalmente ao longo dos afluentes do médio curso do rio Chapecó. Na bacia do rio Irani, que reúne cerca de 109.000 habitantes, existem 8 núcleos urbanos, onde residem em torno de 87.000 habitantes, com destaque para a cidade de Xanxerê. Deve-se destacar na região os processos erosivos dos solos, o que leva à alta turbidez, como se observa no rio Chapecó.

A RH 3 ocupa uma área total de 7.897 km2, e suas principais bacias hidrográficas são as do rio do Peixe e do rio Jacutinga. O rio do Peixe, cuja bacia é de $5.240 \mathrm{~km} 2$, nasce no município de Calmon, zona central de Santa Catarina, e percorre cerca de $290 \mathrm{~km}$ até sua foz no rio Uruguai. Nesse trajeto, recebe as águas dos rios Preto, São Pedro e Santo Antônio pela margem direita, e rios Bonito e Leão pela margem esquerda; $O$ rio Jacutinga nasce na vertente oposta do rio Irani, tendo sua foz situada no lago formado pela barragem de Itá, tem uma área de drenagem de $1.008 \mathrm{~km}^{2}$. Outros afluentes diretos do rio Uruguai, como o riacho Grande e rio do Engano, também estão situados 
na $\mathrm{RH}$ 3. A bacia do rio do Peixe, com 270.000 habitantes, drena a sede de 24 municípios, que concentram cerca de 220.000 habitantes. Na bacia do rio Jacutinga, com 40.000 habitantes, estão situadas as sedes de 8 municípios num total de 24.000 pessoas, que contribuem com o lançamento de esgotos domésticos nas águas (SDS, 2021).

Esses dados e comparações entre as regiões hidrográficas são importantes e destacam que na região hidrográfica com maior número de habitantes o somatório de desastres naturais foi maior mesmo quando comparados com regiões hidrográficas que ocupam maior área, corroborando com Hamdan (2015), Moraes e Borja (2005) e Silva e Rodriguez (2011); isso porque notoriamente os desastres são mais frequentemente comunicados pelos municípios quando afetam a população.

Não deve ser negligenciada questões como treinamento para diagnósticos e registros de desastres, em especial não foram observados registros de desastres naturais biológicos, sendo que é relatado a dengue e Covid-19 (2020). Além disto a fundamental infraestrutura como radar, para monitoramento e sistema de alerta para comunidade.

\section{CONCLUSÃO}

A análise histórica de desastres naturais no período de 1998 a 2019 no estado de Santa Catarina não demonstraram inicialmente uma forte ligação com fenômeno ENSO sobre a hidrologia e meteorologia, e que os desastres naturais são decorrentes das estações de chuva catarinenses, onde ocorre um acumulo de volume de chuvas durante este período. Destacou-se ainda que os maiores registros de ocorrência também estão relacionados ao maior número populacional nas regiões hidrográficas em detrimento até mesmo da área de abrangência de uma bacia hidrográfica.

$O$ estudo pode ser uma ferramenta de monitoramento para visualização das consequências da situação hidrológica, meteorológica e climática no Estado, também pode ser um meio de informação e conscientização da população com dados históricos de eventos, para prevenir e de preparação da população para enfrentamento de desastres naturais. Também poderá auxiliar a execução de políticas públicas de combate aos desastres naturais cíclicos.

No entanto, uma análise estatística deve ser realizada para melhor indicar a contribuição do ENSO nos desastres naturais do estado de Santa Catarina. Bem como deve ser considerado para trabalhos futuros, crescimento populacional e adensamentos populacionais mais volumosos.

\section{AGRADECIMENTOS}

Instituto Anima, Defesa Civil de Santa Catarina e CAPES.

\section{REFERÊNCIAS}

ACEITUNO, P.: On the functioning of the Southern Oscillation in the South American sector - Part I: Surface climate, Mon. Weather Rev., 116, 505-524, 1988.

ANDRADE A.R., BALDO M.C., NERY J.T. 1999. Variabilidade sazonal da precipitação pluviométrica de Santa Catarina. Acta Scient. Tech., 21:923-928.

BANCO MUNDIAL. Avaliação de Perdas e Danos: Inundações Bruscas em Santa Catarina - novembro de 2018. Brasília. 2012.

BORTOLUZZI C.A. 1987. Esboço geomorfológico de Santa Catarina. In: SILVA L.C.DA, BORTOLUZZI C.A. eds. 1987. Texto explicativo para o mapa geológico do estado de Santa Catarina, E: 1:500.00. Florianópolis: Série Textos Básicos de Geologia e Recursos Minerais de Santa Catarina. N1. Series: Mapas e Cartas Sínteses, 3, Seção Geologia, 3. DNPM/SC. pp 133-167.

BOULANGER, J.-P., LELOUP, J., PENALBA, O., RUSTICUCCI, M., LAFON, F., AND VARGAS, W.: Low-frequency modes of observed precipitation variability over the La Plata Basin, Clim. Dynam., 24, 393-413, 2005.

DEFESA CIVIL, Gestão de Risco de Desastres, file:///C:/ Users/Rachel\%20Magnago/Downloads/Gestao_de_ RISCO_de_desastres_BAIXA.PDF

DEFESA CIVIL, Plataforma online dados de desastres do Estado de Santa Catarina, https://www.defesacivil. sc.gov.br/gestao-risco/decretacao-se-ecp/.

SANTA CATARINA - SECRETARIA DE ESTADO DO DESENVOLVIMENTO ECONÔMICO SUSTENTÁVEL - SDS. Recursos Hídricos de Santa Catarina. https://www.aguas.sc.gov.br/jsmallfib_top/DHRI/ bacias_hidrograficas/bacias_hidrograficas_sc.pdf FEDERAÇÃO DAS INDÚSTRIAS DO ESTADO DE SANTA CATARINA. Plano Sustentabilidade para a competitividade da Indústria Catarinense: relatório de atividades 2018 / Federação das Indústrias do Estado de Santa Catarina - Unidade de Competitividade Industrial. Florianópolis: FIESC, 2018.

FAY, M.; GHESQUIERE, F.; SOLO, T. Natural disasters and the urban poor. World bank reconstruction and development, v. 32, p. 4-7, 2003.

GRIMM, A. M. AND AMBRIZZI, T.: Teleconnections into South America from the Tropics and Extratropics on Interannual to Intraseasonal Timescales, in: Past Climate Variability in South America and Surrounding Regions, edited by: Vimeux, F., Dev. Paleoenviron. Res. 14, 159-191, doi:10.1007/978-90-2672-9_7, Springer 2009. 
GRIMM, A. M., BARROS, V. R., AND DOYLE, M. E.: Climate variability in Southern South America associated with El Niño and La Niña events, J. Climate, 13, 35-58, 2000. HAMDAN, F. Intensive and extensive disaster risk drivers and interactions with recent trends in the global political economy, with special emphasis on rentier states. International Journal of Disaster Risk Reduction, v. 14, p. 273-289, 2015.

MARCATTO, T. I.; LIMA, L. A. Sociedade Contemporânea E O Protocolo De Quioto: O Mundo Em Prol Do Meio Ambiente. Connexio - Issn 2236-8760, v. 2, n. 2, p. 4163, 2013.

MENDONCA, M. B. DE; SILVA ROSA, T. DA; BELLO, A. $R$. Transversal integration of geohydrological risks in an elementary school in Brazil: A disaster education experiment. International Journal of Disaster Risk Reduction, v. 39, n. June, p. 101213, 2019.

PARIZZI, M. G. Desastres Naturais e Induzidos e o Risco Urbano. Geonomos. v. 22, p. 1-9, issn: 0104-4486, 2014. MORAES L.R.S.; BORJA P.C. Política e plano municipal de saneamento ambiental: experiências e recomendações. Brasília: Organização Panamericana da Saúde; Ministério das Cidades, Programa de Modernização do Setor de Saneamento, 2005, 135 p.

MOURA, MARKS MELO; et al. Relation of El Niño and La Niña phenomena to precipitation, evapotranspiration and temperature in the Amazon basin. Science of The Total Environment. Volume 651, Part 1, 15 February 2019, Pages 1639-1651.

NOAA. Plataforma online da Administração Oceânica e Atmosférica Nacional. https://origin.cpc.ncep. noaa.gov/products/analysis_monitoring/ensostuff/ ONI_v5.php.

OLIVEIRA, F. R. DE; OURIQUES, J. M. DE A.; CORREIA, L. S. territorium 25 (II), 2018, 5-18. v. 25, n. Iv, p. 5-18, 2018. PENALBA, OLGA C; BELTRAN, ADRIANA; MESSINA, CARLOS. Monthly Rainfall In Central-Eastern Argentina and ENSO: A Comparative Study of Rainfall Forecast Methodologies. REVISTA BRASILEIRA DE AGROMETEOROLOGIA, [S. I.], ano 2005, v. 13, n. 2, p. 49-61, 23 maio 2005.

PENALBA, OLGA CLORINDA; RIVERA, JUAN ANTONIO. Precipitation response to El Niño/La Niña events in Southern South America - emphasis in regional drought occurrences. Adv. Geosci., 42, 1-14, 2016. https:// doi.org/10.5194/adgeo-42-1-2016

ROPELEWSKI, C. F.; HALPERT, M. S.: North American precipitation and temperature patterns associated with the El Niño/Southern Oscillation (ENSO), Mon.
Weather Rev., 114, 2352-2362, 1986. Ropelewski, C. F. and Halpert, M. S.: Global and regional scale precipitation patterns associated with the El Niño/Southern Oscillation, Mon. Weather Rev., 115, 1606-1626, 1987.

SILVA. E. V E; RODRIGUEZ, J. M. M. Geoecologia da Paisagem: Zoneamento e Gestão Ambiental em Ambientes Úmidos e Subúmidos. Revista Geográfica de América Central. Número Especial EGAL, 2011.

SILVA, P. D. V. et al. Planos Familiares De Emergência: Iniciativas Em Curso Em Santa Catarina. Revista Gestão \& Sustentabilidade Ambiental, v. 7, p. 379, 2018.

SHORT A. D.; KLEIN, A. H. D. F. 2016. Brazilian Beach Systems. USA: Springer International Publishing. 611p. SOUZA, C.R. de G.; SOUZA FILHO, P.W.M.; ESTEVES, SL.; VITAL, H. DILLENBURG, S.R.; PATCHINEELAM, S.M. \& ADDAD, J.E. 2005. Praias Arenosas e Erosão Costeira. In: C.R. de G. Souza et al. (eds.). Quaternário do Brasil. Holos, Editora, Ribeirão Preto (SP). p. 130-152.

SOUZA, CEZARINA MARIA NOBRE. Participação dos cidadãos e saneamento básico: panorama da legislação nacional. Revista do Instituto de Estudo Brasileiros USP; n 63; abr 2016; p. 141-158.

TOMINAGA, L. K.; SANTORO, J.; AMARAL, R. Desastres Naturais: conhecer para prevenir. [s.l: s.n.].

TRENBERTH, K. E.; STEPANIAK, D. P.: Indices of El Niño evolution, J. Climate, 14, 1697-1701, 2001.

United Nations Office for Disaster Risk Reduction, https://www.preventionweb.net/organizations/1171 


\section{AUTORES}

ORCID: https://orcid.org/0000-0001-7306-7984

RACHEL FAVERZANI MAGNAGO | Universidade do Sul de Santa Catarina | Mestranda em Ciências ambientais| Palhoça, SC - Brasil | Correspondência para: Dunas da Joaquina, 275, Rio Tavares. - Florianópolis - SC - Brasil, CEP 88048424

ORCID: https://orcid.org/0000-0001-8258-3761

PATRICIA MEDEIROS | Universidade do Sul de Santa Catarina | Mestranda em Ciências ambientais| Palhoça, SC - Brasil | Correspondência para: R. Francisco Jose de Silva 325 Guarda do Cubatão , Palhoça - SC, 88135 822) | e-mail: patriciamedeiros.unisul@gmail.com

ORCID: https://orcid.org/0000-0002-0373-9711

RODRIGO PAULO RAIMUNDO|IFSC|Eng. Elétrica|Florianópolis, SC - Brasil| Correspondência para: Rua Capri, 432 - Pagani - Palhoça/ SC. Cep: 88.132-299| e-mail: rodrigopauloraimundo@gmail.com

ORCID: https://orcid.org/0000-0002-1775-1826

SUSANA CLAUDETE COSTA, M.Sc. | Universidade do Sul de Santa Catarina| Curso | Palhoça, SC - Brasil| Correspondência para: Rua Capri 423 - ap. 603 Cep: 88.132-299| e-mail: Susanacostah@hotmail.com

\section{COMO CITAR ESTE ARTIGO}

MAGNAGO, Rachel Faverzani; MEDEIROS, Patrícia; RAIMUNDO, Rodrigo Paulo; COSTA, Susana Claudete. Desastres Naturais No Estado De Santa Catarina - 1998 A 2019. MIX Sustentável, [S.I.], v. 7, n. 4, p. 105-114, set. 2021. ISSN 24473073. Disponível em:<http://www. nexos.ufsc.br/index.php/mixsustentavel>. Acesso em: dia mês. ano. doi:https://doi.org/10.29183/2447-3073. MIX2021.v7.n4.105-114.

DATA DE ENVI0: 15/06/2021

DATA DE ACEITE: 08/06/2021 